\title{
Neutral Lipid Storage Disease Associated with the PNPLA2 Gene: Case Report and Literature Review
}

\author{
Makoto Samukawa ${ }^{a}$ Naoko Nakamurab Makito Hirano ${ }^{a}$ Miyuki Morikawa ${ }^{a}$ \\ Hanami Sakata $^{a}$ Ichizo Nishino $^{c}$ Rumiko Izumi ${ }^{b}$ Naoki Suzuki ${ }^{b}$ \\ Hiroshi Kurodab Kensuke Shigad, e Kazumasa Saigoh ${ }^{\mathrm{a}}$ Masashi Aoki ${ }^{b}$ \\ Susumu Kusunoki ${ }^{\mathrm{a}}$ \\ aDepartment of Neurology, Kindai University, Faculty of Medicine, Osaka, Japan; bepartment of Neurology, \\ Tohoku University Graduate School of Medicine, Sendai, Japan; 'Department of Neuromuscular Research, National \\ Institute of Neuroscience, National Center of Neurology and Psychiatry, Tokyo, Japan; dDepartment of Neurology, \\ Graduate School of Medical Science, Kyoto Prefectural University of Medicine, Kyoto, Japan; 'Department of \\ Neurology, Matsushita Memorial Hospital, Osaka, Japan
}

\section{Keywords}

Neutral lipid storage disease with myopathy · Triglyceride deposit cardiomyovasculopathy PNPLA2 - Genotypephenotype correlation · Glaucoma · Pulmonary cysts

\begin{abstract}
Mutations in the PNPLA2 gene cause neutral lipid storage disease with myopathy (NLSDM) or triglyceride deposit cardiomyovasculopathy. We report a detailed case study of a 53-year-old man with NLSDM. The PNPLA2 gene was analyzed according to the reported method. We summarized the clinical, laboratory, and genetic information of 56 patients, including our patient and 55 other reported patients with homozygous or compound heterozygous mutations in the PNPLA2 gene. We found a novel homozygous mutation (c.194delC) in the PNPLA2 gene that resulted in frameshift. The patient suffered from normal-tension glaucoma and pulmonary cysts, symptoms that are relatively common in
\end{abstract}

\begin{tabular}{ll}
\hline karger@karger.com & (c) 2020 The Author(s) \\
www.karger.com/ene & Published by S. Karger AG, Basel \\
& This article is licensed under the Creative Commons Attribution- \\
Narger & Nonmercial-NoDerivatives 4.0 International License (CC BY- \\
NC-ND) (http://www.karger.com/Services/OpenAccessLicense). \\
Usage and distribution for commercial purposes as well as any dis- \\
tribution of modified material requires written permission.
\end{tabular}

the elderly but were not previously reported for this disease. Our summary confirmed that Jordan's anomaly, polymorphonuclear leukocytes with lipid accumulation, was the most consistent finding of this disease. Because this disease is potentially treatable, our results may help rapid and correct diagnosis.

(C) 2020 The Author(s)

Published by S. Karger AG, Basel

\section{Introduction}

Neutral lipid storage disease with myopathy (NLSDM) or triglyceride deposit cardiomyovasculopathy (TGCV) is a rare disease because only 7 patients in Japan and 55 patients in the world have been reported in the Englishwritten literature [1-7]. NLSDM/TGCV is caused by mutations in the patatin-like phospholipase domain-containing 2 (PNPLA2) gene, which encodes adipose triglyceride lipase, the rate-limiting enzyme for hydrolysis of 
intracellular triglyceride (TG) [8]. We found a novel mutation in the PNPLA2 gene in a patient with NLSDM and summarized clinical, laboratory, and genetic information of 56 patients, including our patient and 55 reported patients.

\section{Patients and Methods}

\section{Patient Characteristics}

A 53-year-old right-handed man was born to healthy consanguineous parents of Japanese origin with no family history of NLSDM. He showed normal motor and mental development during his growing years. His height was $163.9 \mathrm{~cm}$, and his weight was 52 $\mathrm{kg}$. He smoked 15 cigarettes a day for more than 20 years but quit smoking at the age of 53. At the age of 30 , he exhibited a high level of creatine kinase [9] without any particular symptoms associated with NLSDM. Routine examination of the heart revealed no abnormalities at that time. At the age of 35, he visited the local hospital seeking treatment for atrophy in his right upper arm. Neurological examination revealed the predominant involvement of the right upper limb, with preserved lower limb strength. At the age of 45 , he suffered from a pelvic abscess caused by perforated diverticulitis and underwent surgery for the same. At the time of the surgery, the rectus abdominis muscle was biopsied, and the result was positive for lipid storage disease. The serum carnitine levels were normal. He was referred to our hospital at the age of 47 .
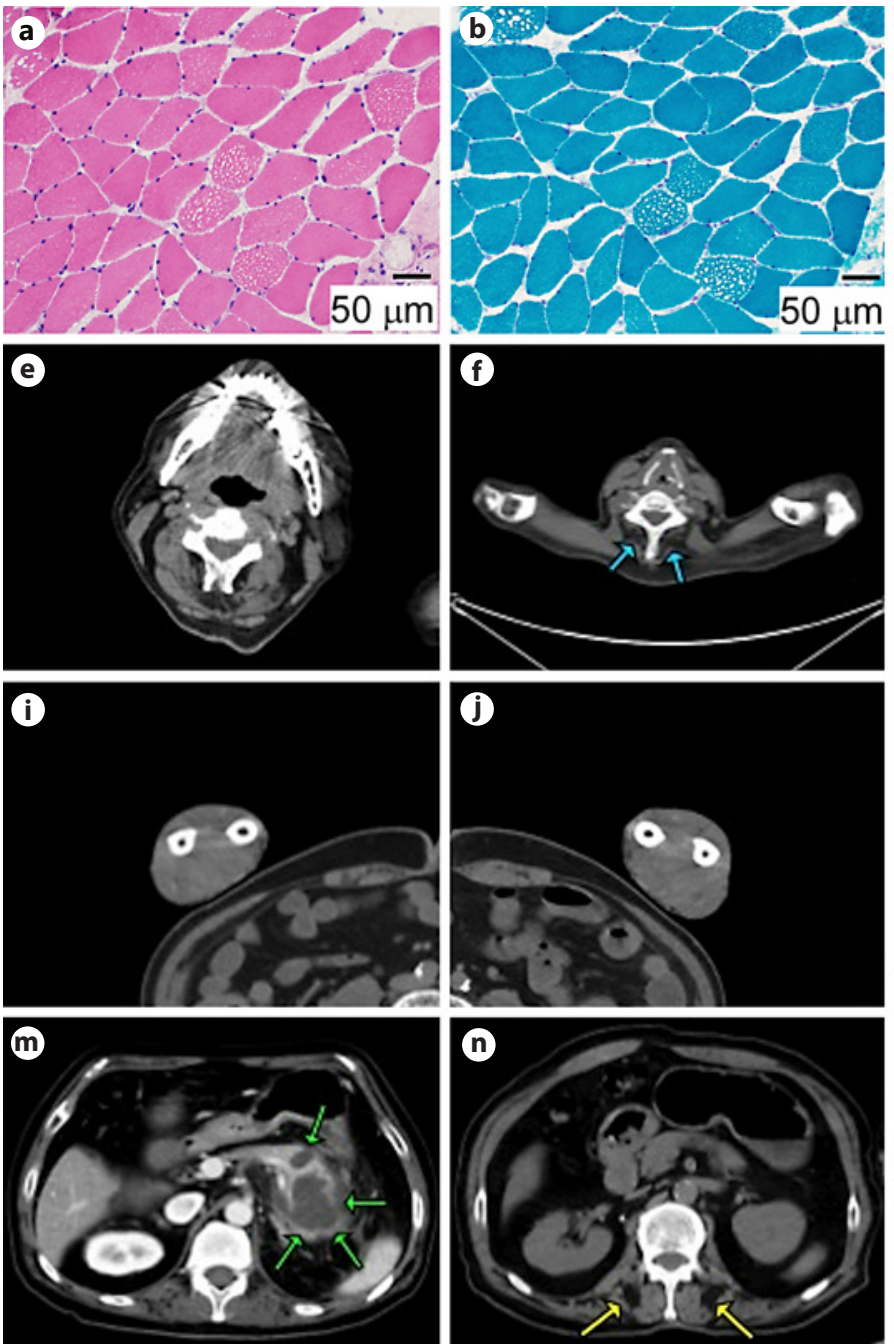

Fig. 1. Clinical and imaging information of our patient with a mutation in the PNPLA2 gene. a-c Muscle histopathology. a H\&E staining showed myofibers of varying sizes with marked vacuolation. Scale bar $=50 \mu \mathrm{m}$. $\mathbf{b}$ These vacuoles are not rimmed, as confirmed by modified Gomori trichrome stain. c The vacuoles contained lipids, as observed by positive Oil Red O staining. High-powered view of the area within the black square (inset).
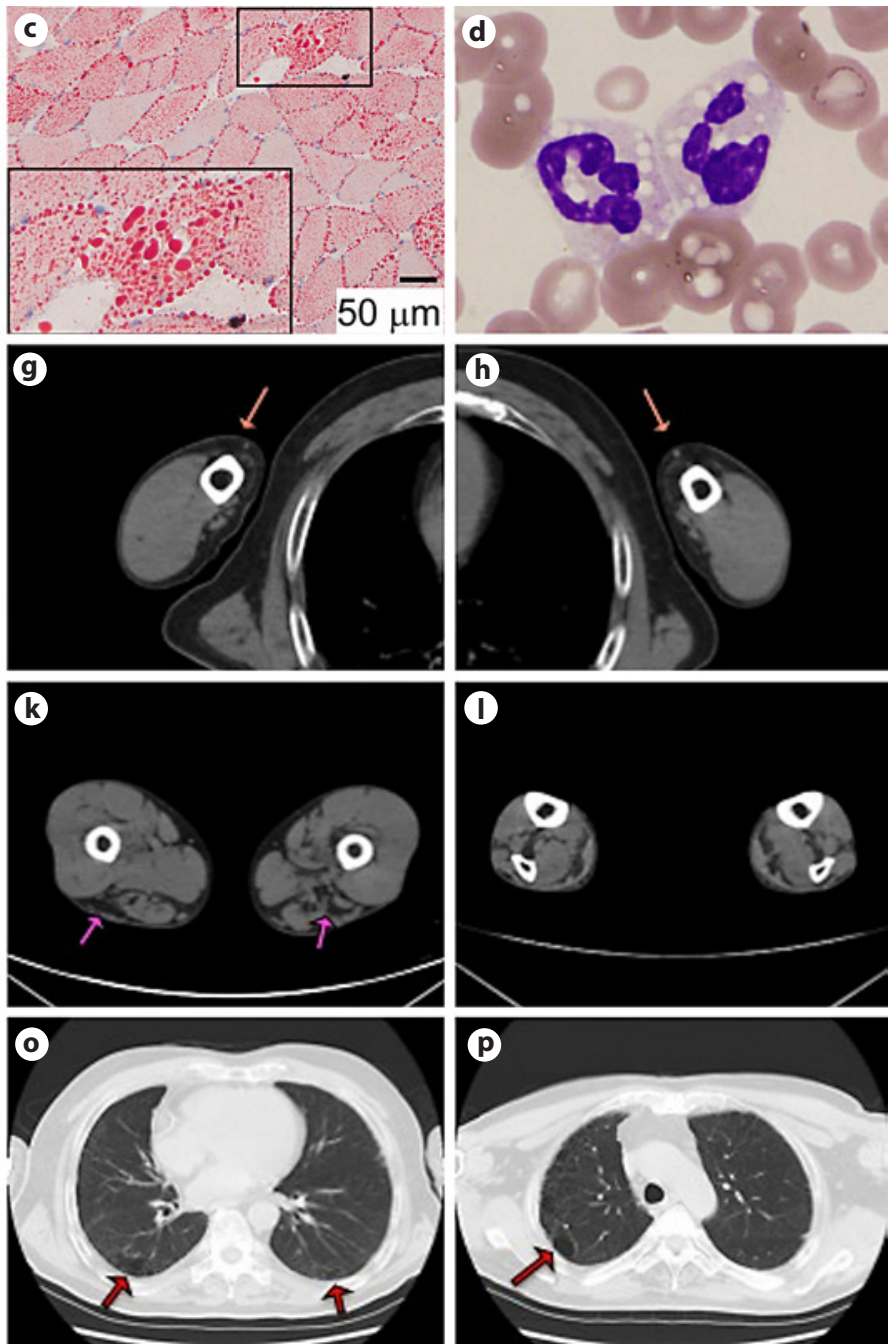

d Peripheral blood smear showing the presence of lipid droplets in the patient's leukocytes (Jordan's anomaly). e-i, $\mathbf{n}$ Skeletal muscle CT showing atrophy in the paraspinal muscles (blue and yellow arrows), biceps brachii muscles (orange arrow), and posterior muscles in the lower limbs (red arrow). $\mathbf{m}$ CT showing pancreatitis with cysts (green arrow). $\mathbf{n}$ Recent CT showing atrophy of the pancreas. $\mathbf{o}, \mathbf{p}$ CT showing bilateral pulmonary cysts (large arrow). 
The muscle weakness had spread to the left upper limb and then to the lower limbs as well. At 50 years of age, ECG showed mild ST elevation. He suffered from acute pancreatitis and developed a pancreatic cyst. CT showed presence of bilateral pulmonary cysts without any symptoms. At the age of 51, clumsiness of the right hand became apparent, whereas at the age of 53 years, he experienced difficulties in walking upstairs and in swallowing. In addition, weakness in his left arm progressed gradually. He exhibited no skin abnormalities. Ophthalmological examinations revealed normal-tension/open-angle glaucoma ( $\mathrm{rt}, 13 \mathrm{~mm} \mathrm{Hg}$ and lt, 13 $\mathrm{mm} \mathrm{Hg}$ ), whereas his visual acuity was normal (rt, 0.9 and lt, 0.9). He was then admitted to our hospital for re-evaluation. Neurological examinations revealed severe weakness of the proximal muscles of the upper limbs on both sides. Weakness in the lower limbs included mild weakness in the bilateral tibialis anterior muscle, whereas relatively preserved strength in other muscles was observed. Deep tendon reflexes bilaterally decreased at the biceps brachii, brachioradialis, and Achilles tendon but were preserved at the triceps and patellar tendon. Sensory and autonomic functions were unaltered. Laboratory data showed increased CK and TG. As the $\mathrm{HbA} 1 \mathrm{c}$ levels increased to $10 \%$, he needed insulin therapy. His muscle biopsy findings, Jordan's anomaly, and CT scans for muscle, abdominal, and pulmonary regions are shown in Figure 1.

\section{Ethics}

Patients gave their written informed consent for their gene analyses and publication of the article (including imaging results). The study protocol was approved by the Institutional Review Board of Kindai University and Tohoku University on human research, as reported previously $[6,8]$.

\section{RNA Analyses}

Total RNA was extracted from muscles of control, the current patient (Pt1), and a previously reported patient (Pt2) with
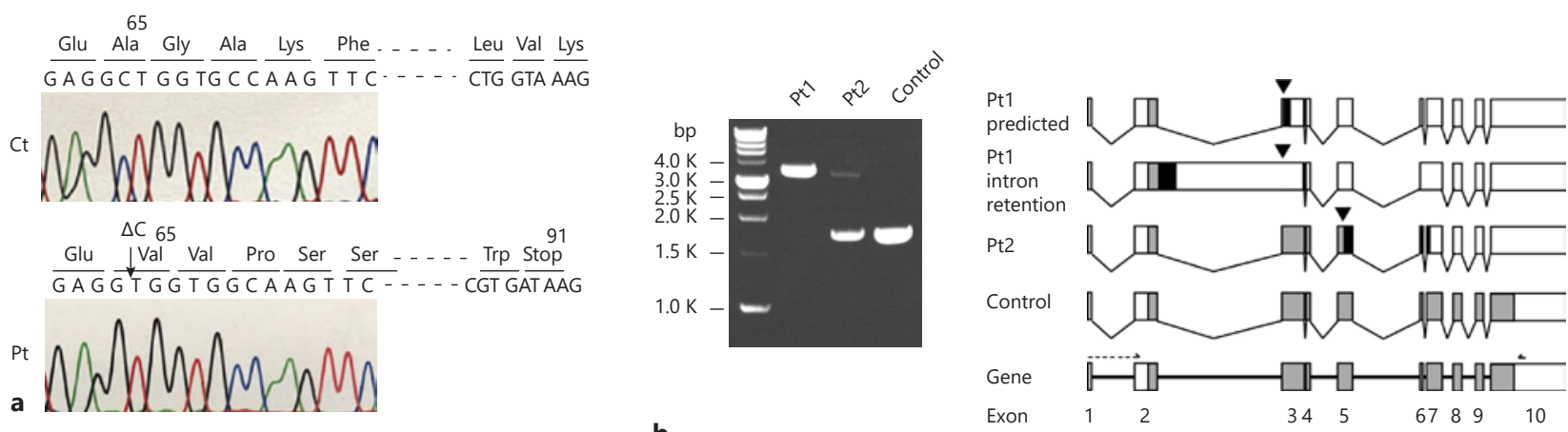

b

Hydrophobic

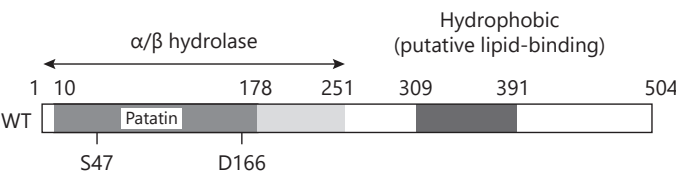

Pt1

predicted

Pt1

intron

retention

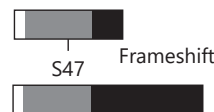

D166

S47 Frameshift

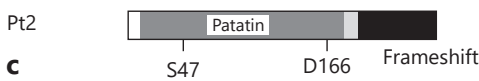

$\begin{array}{llll}\text { C } & \text { S47 } & \text { D166 } & \text { Frameshift }\end{array}$

Fig. 2. Genetic results. a Sanger sequencing showing that the patient exhibited a deletion of C (c.194delC) at codon 65, as compared to sequences of control. This mutation is predicted to cause a frameshift and termination at codon 91. b RT-PCR analyses of the muscle RNA showed a larger product in Pt1 than in control. Pt2 with c.576delC (p.Asp192Glufs*64) in exon 5 had a major product with the same size as that of Ct. Pt2 also had a minor transcript with the same size as that of Pt1. The top of the right panel shows a transcript of Pt1 predicted by genomic mutation as described in panel A, which is not present in the left panel of $\mathbf{b}$. The second transcript of Pt1 has been detected in the left panel, with retention of introns 2 and 6. The major transcript of Pt 2 shows a transcript as predicted by genomic mutation. Pt2 also had a transcript with retention of introns 2 and 6 as in Pt1. Closed triangle, deletion mutation. Gray area, coding region. Closed area, frameshifted region. Arrow, primer position used for RT-PCR. c Structures of WT and mutant ATGL protein encoded by the PNPLA2 gene. A predicted transcript or a transcript identified by RT-PCR in Pt1 has the loss of half of the patatin-like domain including D166 but preserves the $S 47$ catalytic site. The patatin-like domain is preserved in Pt2. Pt1, current patient; Pt2, previously reported patient; Ct, control; WT, wild-type; ATGL, adipose triglyceride lipase; D166, catalytic site Asp166; S47, Ser47. 
Table 1. Information of patients with homozygous or compound heterozygous mutations in the PNPLA2 gene

\begin{tabular}{|c|c|c|c|c|c|}
\hline & $\begin{array}{l}\text { This } \\
\text { report }\end{array}$ & $\begin{array}{l}\text { Positive } \\
\text { patients }\end{array}$ & & $\begin{array}{l}\text { Examined } \\
\text { patients }\end{array}$ & $\begin{array}{l}\text { Percentage of } \\
\text { positive patients }\end{array}$ \\
\hline \multicolumn{6}{|l|}{ Backgrounds } \\
\hline Gender, male & + & 32 & l & 61 & 52 \\
\hline Consanguinity & + & 19 & l & 43 & 44 \\
\hline Origin, Japan & + & 8 & l & 61 & 13 \\
\hline \multicolumn{6}{|l|}{ Skeletal myopathy } \\
\hline \multicolumn{6}{|l|}{ Symptoms } \\
\hline Proximal predominant & + & 31 & / & 44 & 70 \\
\hline Distal predominant & - & 8 & l & 44 & 18 \\
\hline Proximal = distal & - & 5 & l & 44 & 11 \\
\hline Asymmetric & + & 30 & / & 38 & 79 \\
\hline Right $>$ left & + & 25 & l & 30 & 83 \\
\hline Left $>$ right & - & 1 & l & 30 & 3 \\
\hline \multicolumn{6}{|l|}{ Muscle biopsy } \\
\hline Lipid accumulation & + & 52 & l & 53 & 98 \\
\hline Rimmed vacuoles & - & 11 & l & 53 & 21 \\
\hline Cardiomyopathy & + & 30 & / & 56 & 54 \\
\hline Hyperlipidemia & - & 17 & l & 47 & 36 \\
\hline Diabetes mellitus & + & 13 & l & 44 & 30 \\
\hline Pancreatitis & $+($ acute $)$ & 6 & l & 36 & 17 \\
\hline Hepatomegaly & - & 9 & l & 41 & 22 \\
\hline Splenomegaly & - & 1 & l & 33 & 3 \\
\hline Mental impairment & - & 3 & l & 43 & 7 \\
\hline Ophthalmopathy & + , glaucoma & 1 & l & 32 & 3 \\
\hline Hearing impairment & + & 9 & l & 53 & 17 \\
\hline Skin disorders & - & 3 & l & 46 & 7 \\
\hline Skeletal disorders & - & 11 & l & 44 & 25 \\
\hline Jordans' anomaly & + & 50 & l & 50 & 100 \\
\hline
\end{tabular}

Italic percentages indicate that parameters decrease by $10 \%$ or more than those reported previously; bold percentages indicate that parameters increase by $10 \%$ or more than those reported previously or that those are newly detected in this study.

c.576delC (p.Asp192Glufs*64) in exon 5 [6], and reverse-transcribed with oligo dT primer, as described previously $[10,11]$. The primers set to cover the entire coding sequence were PNPLA2_-168F: 5'-AGGCAGGGCCAGACCCAGCTTCTT-3' and PNPLA2_1605R: 5'-GACCCCTCTTGGCAACTGTGAGTC-3'. Primers used for sequencing were PNPLA2-543R $5^{\prime}$-GGTGTTCTTAAGCTCATAGAGT-3', PNPLA2-562F 5' -TCGGGCGAGAGTGACATCTGTC-3', PNPLA2 658R 5'-AGAGGTTGCGCAGGTTGAACTG-3' PNPLA2-407F 5'-ACGAGCTCATCCAGGCCAATGTC-3', PNPLA2-782R 5'-AAGGGGTTGGGCCGGTTCAGGA-3', PNPLA2-1073F 5' -ACGTTCCCGAGGACATCCGGTGGA-3', and PNPLA2-1145R 5'-TTGGCGCGCATCACCAGGTACTG- $3^{\prime}$. The control muscle was obtained from a woman aged 50 years who had muscle weakness, but the muscle specimens were not apparently altered.

\section{Patient Summary}

We summarized the characteristics of 56 patients, including our patient and 55 reported patients with homozygous or compound heterozygous mutations in the PNPLA2 gene (online suppl. material 1; see www.karger.com/doi/10.1159/000508346 for all online suppl. material). Several authors summarized the characteristics of 37 patients with PNPLA2 gene alterations, where 2 patients with heterozygous symptomatic carriers were included [6]. We excluded the data of these 2 patients from this study.

\section{Results}

\section{Gene Mutation}

The genetic analysis revealed that the patient was homozygous for a deletion mutation (c.194delC) in exon 3 of the PNPLA2 gene (Fig. 2a) that was predicted to result in p.Ala65Valfs*27. This mutation has not been previously reported to our knowledge.

\section{The Result of RNA Analyses}

The current patient had a larger product than control. A previously reported patient with c.576delC (p.Asp- 
192Glufs*64) in exon 5 (Pt2) had a major product with the same size as that of control. Pt2 also had a minor transcript with the same size as that of Pt1. Sequence analyses revealed that the larger product had retention of intron 2 and intron 6 (online suppl. material 2), that encoded a truncated protein with frameshift (p.Ala65Argfs*93). Pt2 had a transcript of retention of introns 2 and 6 and normally spliced transcript with c.576delC in exon 5 .

\section{Summarized Information of Our Patient and Reported Patients}

A summary of patients with homozygous and compound heterozygous mutations in the PNPLA2 gene can be found in Table 1. The table shows that parameters decrease (predominant sites of skeletal myopathy) or increase (consanguinity, asymmetric muscle involvement, and occurrences of cardiomyopathy and hyperlipidemia) by $10 \%$ or more than those reported previously [6]. In addition, a newly detected ophthalmologic abnormality, normal tension glaucoma, is included.

\section{Discussion}

We report a patient homozygous for a novel frameshift mutation in the PNPLA2 gene. The mutant transcript encoded a truncated protein that shared only with 64 amino acids out of 504 amino acids of the adipose triglyceride lipase protein. This indicated that the truncated protein resulted in the loss of more than half of the patatin-like domain, including the catalytic residue Asp166, suggesting the loss of catalytic activity. Because mutations in the PNPLA2 gene are present mainly in exons $4-7$, exon 3 is a relatively rare mutation site.

The reduced amount of transcripts with frameshift mutations is well known as nonsense-mediated mRNA decay [12]. Accordingly, our RNA analyses revealed that a normal-size transcript with the newly identified deletion mutation (c.194delC) was not detected. However, a transcript with a larger size, which contained introns 2 and 6, was unexpectedly identified. Such a transcript seems expressed physiologically, since it has been listed in an expressed sequence tag database in NCBI (AW968685, HY048345, and AW028898). The deletion mutation resides at the seventh base from the splice site and does not generally affect splicing. In addition, this mutation is located far from intron 6. From these facts, we speculate that retention of introns 2 and 6 may occur physiologically, but it may be detected only when a major transcript is reduced in amount. Our speculation may be partly supported by the finding that a previously reported patient with a truncation mutation in exon 5 also had a larger size transcript.

Our patient had normal-tension glaucoma and pulmonary cysts. To date, these symptoms have not been reported for this disease. However, glaucoma is common in the elderly. Pulmonary cysts are also found in smokers, and the patient had a previous history of smoking. Thus, these rare symptoms for NLSDM may have occurred independently of this disease. In addition, he exhibited relatively rare pathological conditions: acute pancreatitis and diabetes. The former occurs in less than $20 \%$ of reported patients, while the latter occurs in $30 \%$.

Our summary revealed that the patient had common features of NLSDM. These included Jordan's anomaly, polymorphonuclear leukocytes with lipid accumulation, which has been consistently found in $100 \%$ of patients to date. Another characteristic symptom is the asymmetrical involvement of limb muscles. A previous study showed that, in this disease, there is predominant involvement of the right upper limb and that the overuse of the dominant right side was possibly associated with the laterality [6]. Of course, it was understandable that the weakness of the dominant side was readily noticed by the patients, compared to the lesser used nondominant side. Thus, further studies are needed to examine this association.

\section{Conclusions}

We report a patient who had atypical symptoms for NLSDM with a new mutation in the PNPLA2 gene. Further studies are necessary to verify that these symptoms are associated with this disease. Our summary demonstrates that Jordan's anomaly is the most consistent finding [13]. Treatment with bezafibrate has been proposed, with no or little clinical benefit in patients $[14,15]$. In contrast, medium-chain triglycerides such as tricaprin improved myocardial function in a TGCV mouse model [16]. Our results may help rapid and correct diagnosis for initiating early treatment.

\section{Acknowledgements}

The authors thank Ms. Naoko Shimakura of Tohoku University for her technical assistance and Dr. Ikuko Mizuta of Graduate School of Medical Science, Kyoto Prefectural University of Medicine, for providing research material. 


\section{Statement of Ethics}

Our patient in this article gave his written informed consent for gene analyses and publication of the article (including imaging results). The study protocol was approved by the institute's committee on human research.

\section{Disclosure Statement}

The authors have no conflicts of interest to declare.

\section{Funding Sources}

This work was supported by the Ministry of Health, Labour and Welfare of Japan (H29-nanchitou [nan]-ippan-030); an Intramural Research Grant (29-4) for Neurological and Psychiatric Disorders of NCNP; and AMED under Grant No. 18kk0205001s0203.

\section{Author Contributions}

(1) Design or conceptualization of the study. (2) Acquisition of the data. (3) Analysis or interpretation of the data. (4) Drafting or revising the manuscript for intellectual content. Makoto Samukawa has contributed to 1,2, and 3. Naoko Nakamura has contributed to 2 and 3. Makito Hirano has contributed to 2, 3, and 4. $\mathrm{Mi}$ yuki Morikawa has contributed to 2, 3, and 4. Hanami Sakata has contributed to 2, 3, and 4. Ichizo Nishino has contributed to 2, 3, and 4. Rumiko Izumi has contributed to 2, 3, and 4. Naoki Suzuki has contributed to 2, 3, and 4. Hiroshi Kuroda has contributed to 2, 3, and 4. Kensuke Shiga has contributed to 2, 3, and 4. Masashi Aoki has contributed to 1, 3, and 4. Susumu Kusunoki has contributed to 1,3 , and 4 .

\section{References}

1 Oshima $Y$, Hirota $H$, Nagai H, Izumi M, Nakaoka Y, Osugi T, et al. Images in cardiovascular medicine. specific cardiomyopathy caused by multisystemic lipid storage in Jordan's anomaly. Circulation. 2002 Jul 9;106(2): 280-1.

2 Akiyama M, Sakai K, Ogawa M, McMillan JR, Sawamura D, Shimizu H. Novel duplication mutation in the patatin domain of adipose triglyceride lipase (PNPLA2) in neutral lipid storage disease with severe myopathy. Muscle Nerve. 2007 Dec;36(6):856-9.

3 Kobayashi K, Inoguchi T, Maeda Y, Nakashima N, Kuwano A, Eto E, et al. The lack of the Cterminal domain of adipose triglyceride lipase causes neutral lipid storage disease through impaired interactions with lipid droplets. J Clin Endocrinol Metab. 2008 Jul;93(7):2877-84.

4 Ohkuma A, Nonaka I, Malicdan MC, Noguchi S, Ohji S, Nomura K, et al. Distal lipid storage myopathy due to PNPLA2 mutation. Neuromuscul Disord. 2008 Aug;18(8):671-4.

5 Laforêt P, Vianey-Saban C. Disorders of muscle lipid metabolism: diagnostic and therapeutic challenges. Neuromuscul Disord. 2010 Nov;20(11):693-700.

6 Kaneko K, Kuroda H, Izumi R, Tateyama M, Kato M, Sugimura K, et al. A novel mutation in PNPLA2 causes neutral lipid storage disease with myopathy and triglyceride deposit cardiomyovasculopathy: a case report and literature review. Neuromuscul Disord. 2014 Jul;24(7):634-41.

7 Latimer CS, Schleit J, Reynolds A, Marshall DA, Podemski B, Wang LH, et al. Neutral lipid storage disease with myopathy: further phenotypic characterization of a rare PNPLA2 variant. Neuromuscul Disord. 2018 Jul; 28(7):606-9.

8 Fischer J, Lefèvre C, Morava E, Mussini JM, Laforêt P, Negre-Salvayre A, et al. The gene encoding adipose triglyceride lipase (PNPLA2) is mutated in neutral lipid storage disease with myopathy. Nat Genet. 2007 Jan; 39(1):28-30.

9 Xie X, Langlais P, Zhang X, Heckmann BL, Saarinen AM, Mandarino LJ, et al. Identification of a novel phosphorylation site in adipose triglyceride lipase as a regulator of lipid droplet localization. Am J Physiol Endocrinol Metab. 2014 Jun 15;306(12):E1449-59.

10 Hirano M, Yamamoto A, Mori T, Lan L, Iwamoto TA, Aoki M, et al. DNA single-strand break repair is impaired in aprataxin-related ataxia. Ann Neurol. 2007 Feb;61(2):162-74.

11 Hirano M, Samukawa M, Isono C, Saigoh K, Nakamura Y, Kusunoki S. Noncoding repeat expansions for ALS in Japan are associated with the ATXN8OS gene. Neurol Genet. 2018 Aug;4(4):e252.
12 Hirano M, Hung WY, Cole N, Azim AC, Deng HX, Siddique T. Multiple transcripts of the human $\mathrm{Cu}, \mathrm{Zn}$ superoxide dismutase gene. Biochem Biophys Res Commun. 2000 Sep 16; 276(1):52-6.

13 Suzuki A, Nagasaka H, Ochi Y, Kobayashi K, Nakamura H, Nakatani D, et al. Peripheral leukocyte anomaly detected with routine automated hematology analyzer sensitive to adipose triglyceride lipase deficiency manifesting neutral lipid storage disease with myopathy/triglyceride deposit cardiomyovasculopathy. Mol Genet Metab Rep. 2014; 1:249-53.

14 van de Weijer T, Havekes B, Bilet L, Hoeks J, Sparks L, Bosma M, et al. Effects of bezafibrate treatment in a patient and a carrier with mutations in the PNPLA2 gene, causing neutral lipid storage disease with myopathy. Circ Res. 2013 Mar 1;112(5):e51-4.

15 Garcia MA, Rojas JA, Millán SP, Flórez AA Neutral lipid storage disease with myopathy and dropped head syndrome. report of a new variant susceptible of treatment with late diagnosis. J Clin Neurosci. 2018 Dec;58:207-9.

16 Suzuki A, Yamaguchi S, Li M, Hara Y, Miyauchi $\mathrm{H}$, Ikeda $\mathrm{Y}$, et al. Tricaprin rescues myocardial abnormality in a mouse model of triglyceride deposit cardiomyovasculopathy. J Oleo Sci. 2018 Aug 1;67(8):983-9. 